\title{
Domain Separation with Port Interfaces for Calculation of Emissions from Enclosure Slots
}

\author{
Christian Poschalko \\ Diesel \& Gasoline Systems, Robert Bosch AG \\ Vienna, Austria
}

\begin{abstract}
An equivalent circuit method is used to define ports at the slots of enclosures to separate the external field domain from the internal over a broad frequency range. The aspect ratios of internal to external structures of electronic devices are usually difficult to handle in joint domain simulations. Our method provides the opportunity to simulate the domains separately and to connect the two models afterwards. We present an example for a cubical enclosure with a slot. We use a cavity model and an impedance network at the slot to consider the radiation loss. The results are compared with HFSS $^{\circledR}$ simulations.
\end{abstract}

Keywords - domain separation, electrically equipped enclosures, electromagnetic compatibility, radiated emissions

\section{INTRODUCTION}

The internal structures of electronic devices are often fine and dense (geometric structures in $\mathrm{mm}$ to $\mathrm{cm}$ range), whereas the external structures can have large extensions (m range). This is difficult to handle for simulation programs, because the required meshes rapidly exceed memory and simulation time limitations in practical applications. An example for a very complex simulation problem is a device with a cable harness and multiple scattering objects in the external environment. However, not only the complexity is a problem, a further disadvantage of a joint domain simulation is that external influences cannot be separated from the internal ones. When a device is designed, the EMC designer wants to optimize the device independent of the external environment, especially, if it is intended for the use in different external environments. Therefore, it is necessary to know the internal influences which can be controlled by the design, whereas the external influences cannot be controlled in most applications.

To separate the field calculation of the internal and the external domain of a device with a metallic enclosure with slots, we introduce ports at the slots, which can be used to connect the enclosure to any external environment. The external environment can then be simulated with ports at the same slot positions. This approach allows to separate the domains and to evaluate the behavior of the device in different environmental situations.

\author{
Siegfried Selberherr \\ Institute for Microelectronics, Technische Universität Wien \\ Vienna, Austria
}

In Section II we present the separation approach using the equivalent source method to define the interface ports. We introduce a PEM (perfect magnetic conducting surface) at the slot face of the enclosure to separate the internal domain of the device from the external. Then we place excitation ports on both sides of the PEM surface and perform two separate simulations, one with the internal ports active (the external inactive) and the other with the external ports active (the internal inactive). Two network models are thereby obtained, which can be simulated together in a network simulation program.

As an application of the method, we calculate the transfer impedance from a port inside a slim cubical enclosure to a port at the slot (Fig. 1). We use a cavity field model to calculate the internal field of the device. This cavity field model is developed with a PEM boundary condition and ports at the slot to establish an interface, which enables the connection of a network (model of the external domain). This is the same separation approach as we describe in Section II.

For the model for the external environment we use ports at the slot (outside of the enclosure, in front of a PEM surface at the slot). We use an analytical far field expression, to obtain a network model for the external environment, which is connected to the ports of the previously calculated internal enclosure model. This external model introduces the radiation loss to the lossless cavity model of the internal enclosure domain. The results are compared with $\mathrm{HFSS}^{\circledR}$ simulations, which have been done using a model with an open slot. The comparison shows a good agreement to the cavity model calculation with the separation approach.

Section III contains the cavity model expressions for the internal of a slim cubical enclosure. We obtained this formulation similarly as it has been done for power planes by [1] and [2] but with a variation of the boundary condition to consider the closed metallic walls of the enclosure. We also present the impedance matrix for the consideration of the external environment in Section III, which we obtain by the use of an equivalent source approach [3][4][6][7] . 
In Section IV we present the comparison of this calculation with the $\mathrm{HFSS}^{\circledR}$ simulation results. A comparison of the $\mathrm{HFSS}^{\circledR}$ results to the cavity model results without the consideration of the radiation loss from the external impedance network shows that this radiation loss has a significant influence on the internal cavity fields. [5] has shown that this influence is important for power planes with a high plane separation distance. As the plane separation in case of an enclosure is much higher than that of power planes, the radiation loss becomes the significant loss mechanism. We use the cavity model to present the radiated power from the slot of the enclosure, depending on the positions of sources inside the enclosure. The approach with the introduction of the external environment and the radiation loss by ports and an impedance matrix is very useful for this application. [5] calculated the internal cavity fields of power planes and used these results to calculate the radiation loss, which makes it necessary to calculate the radiation loss for every internal source situation. Since the impedance matrix in our method is independent of the internal source configuration, this matrix can be used for any source situation.

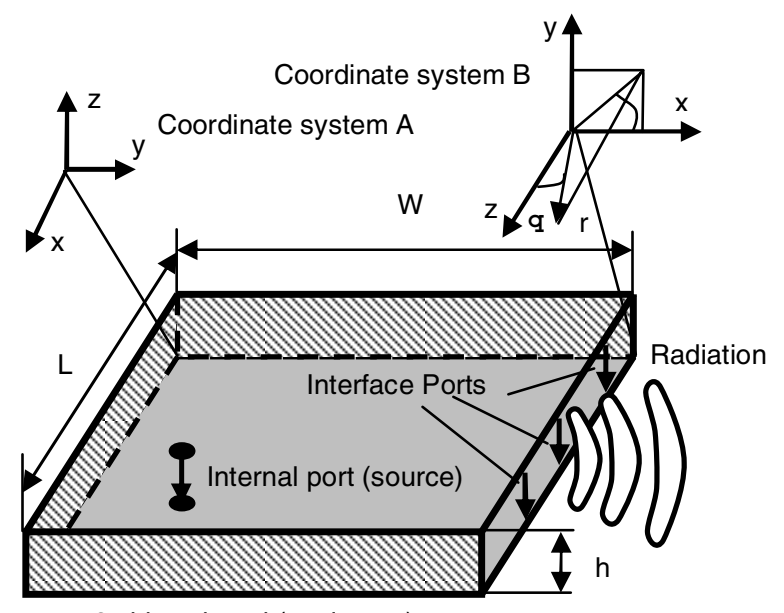

3 sides closed (enclosure)

Figure 1. A cubical metallic enclosure with three closed edges and one open slot. The cavity field inside the enclosure causes radiated emission from the open slot. Coordinate system A is issued for the derivation of the cavity field expression, Coordinate system B for the impedance matrix calculation to consider the external environment (radiation loss).

\section{DOMAIN SEPERATION BY PORTS AND PEM SURFACES}

Excitation ports are usually defined with a quasi static approach. In this case the ports have to be electrically short compared to the wavelength in order to get correct simulation results. Slots inside metallic enclosures have usually one geometric dimension which is small compared to the wavelength. In Fig. 1 this is the $\mathrm{z}$ dimension (Coordinate system A). Therefore it is possible to define excitation ports at the slot. However, these ports excite the interior and the exterior of the device and separation of the domains is not possible.
To separate the internal domain from the external, we introduce a PEM condition on the slot face in front of current source ports. When the ports are placed outside the enclosure just in front of the PEM wall, they act like current sources and enforce a magnetic field. The impedance matrix calculated with this method is a model for the external environment, which is independent of the interior of the device. When the excitation ports are placed on the other side of the PEM wall inside the enclosure, they are used to calculate a model of the interior, which can be connected to the external impedance network afterwards. Fig. 2 shows that this approach is consistent with the equivalent source theory.
$\mathrm{E}, \mathrm{H}$

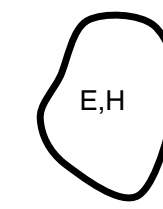

E,H

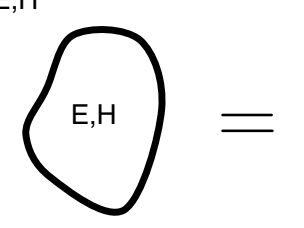

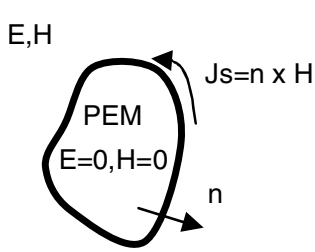

$\mathrm{E}, \mathrm{H}$

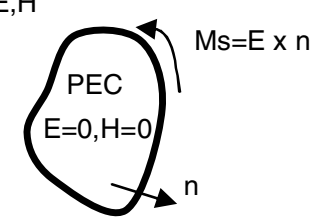

Figure 2. Equivalent source theory. Electric current sources and a PEM boundary condition on the surface of an obstacle cause the same fields. Magnetic current sources and a PEC (perfectly electric conducting) cause also the same fields.

When the currents in front of the PEM surface are forced by ports, an impedance matrix can be calculated which will be correct for any source at these ports. A port definition at the opposite side of the PEM wall gives a model for the other domain. Both models are independent of each other and support the connection of an arbitrary network at the interface ports. The models can be used for an individual optimization of the separated device domain and for the prediction of the behavior of the device in different environments. Such a port definition can easily be carried out in a three-dimensional full wave simulation tool such as $\operatorname{HFSS}^{\circledR}\left(\right.$ Ansoft $\left.^{\circledR}\right)$. A similar method would also be possible, namely using PEC walls and magnetic current ports (voltage ports), as shown in Fig. 2. We have only used PEM boundary surfaces and current ports, because an open slot behaves more like a PEM wall than a PEC wall.

In the following section we present an analytical formulation for the cavity fields, which we use in Section IV together with this separation method to calculate the transfer impedance of internal ports to ports at the connector, considering the radiation loss, introduces by a model of the external environment. 


\section{CAVITY Model AND ELECTRIC FAR FIELD EXPRESSION FOR A SLIM CUBICAL ENCLOSURE}

We derived the cavity field formulations within the enclosure depicted in Fig. 1 by using the scalar Helmholz equation (1) similarly as it has been done for rectangular planes with open boundaries by [1][2].

$$
\vec{\nabla}^{2} E_{z}+\omega^{2} \cdot \mu_{0} \cdot \varepsilon_{0} \cdot E_{z}=j \cdot \omega \cdot \mu \cdot J_{z}
$$

\section{$E_{z} \quad z$ component of the electric field (between the planes) $J_{z} \quad z$ component of the current density}

Using the separation method and introducing PEC boundary conditions for the closed walls and a PEM boundary condition for the open slot, the solution of (1) becomes:

$$
\begin{aligned}
& \mathrm{Z}_{\mathrm{ij}}=\frac{j \cdot \omega \cdot \mu_{0} \cdot h}{L \cdot W} \cdot \sum_{m=0}^{\infty} \sum_{n=0}^{\infty}\left\{\frac{4}{k_{x, m}^{2}+k_{y, m}^{2}-k^{2}} .\right. \\
& \sin \left(k_{x, m} \cdot x_{i}\right) \cdot \sin \left(k_{y, n} \cdot y_{i}\right) . \\
& \left.\sin \left(k_{x, m} \cdot x_{j}\right) \cdot \sin \left(k_{y, n} \cdot y_{j}\right)\right\} \\
& x_{i}, y_{i}, x_{j}, y_{j} \quad \text { port positions of port } i \text { and } j \\
& k_{x, m}=\frac{m \cdot \pi}{L}, \quad k_{y, n}=\frac{(2 \cdot n+1) \cdot \pi}{2 \cdot W}, \quad k=\frac{\omega}{c_{0}}
\end{aligned}
$$

The voltage between the upper and the lower plane at position (xi, yi) is given by:

$$
\begin{gathered}
\mathrm{U}_{\mathrm{i}}\left(x_{i}, y_{i}\right)=\sum_{j=1}^{n}-\text { port }\left(Z_{i j}\left(x_{i}, y_{i}, x_{j}, y_{j}\right) \cdot I_{j}\left(x_{j}, y_{j}\right)\right) \\
I_{j}\left(x_{j}, y_{j}\right) \quad \text { current on port } j \\
n_{-} \text {port } \quad \text { number of ports with } \\
\text { non zero current }
\end{gathered}
$$

The electric field inside the enclosure is given by:

$$
\overrightarrow{\mathrm{E}}_{\mathrm{i}}\left(x_{i}, y_{i}\right)=\frac{U_{i}\left(x_{i}, y_{i}\right)}{h} \cdot \vec{e}_{z}
$$

The resonance frequencies of the enclosure are given by:

$$
f_{r}=\frac{c_{0}}{2 \cdot \pi} \cdot \sqrt{\left(\frac{m \cdot \pi}{L}\right)^{2}+\left(\frac{(2 \cdot n+1) \cdot \pi}{2 \cdot W}\right)^{2}}
$$

This shows that an enclosure with only one open slot has different resonance frequencies as two planes with four open boundaries.

Resonance frequencies of the modes with $\mathrm{m}=0$ do not exist, because the nominator of (2) vanishes at the same frequency as the denominator $(\sin ()$ function). For example, an enclosure with $\mathrm{L}=160 \mathrm{~mm}$ and $\mathrm{W}=120 \mathrm{~mm}$ has the resonance frequencies summarized in Table I:

TABLE I.

RESONANCE FREQUENCIES OF AN ENCLOSURE WITH ONE OPEN EDGE

\begin{tabular}{|c|c|c|c|}
\hline \multicolumn{2}{|c|}{ Mode } & Resonance frequency & Exist \\
\hline $\mathbf{m}$ & $\mathbf{n}$ & $\mathbf{M H z}$ & yes/no \\
\hline 0 & 0 & 625 & no \\
\hline 1 & 0 & 1127 & yes \\
\hline 0 & 1 & 1875 & no \\
\hline 1 & 1 & 2096 & yes \\
\hline
\end{tabular}

Table II contains the resonance frequencies of two planes with $L=160 \mathrm{~mm}, W=120 \mathrm{~mm}$ and four open edges, which were calculated following [1]:

$$
f_{r}=\frac{c_{0}}{2 \cdot \pi} \cdot \sqrt{\left(\frac{m \cdot \pi}{L}\right)^{2}+\left(\frac{n \cdot \pi}{W}\right)^{2}}
$$

TABLE II.

RESONANCE FREQUENCIES OF AN ENCLOSURE WITH FOUR OPEN EDGES

\begin{tabular}{|c|c|c|c|}
\hline \multicolumn{2}{|c|}{ Mode } & Resonance frequency & Exist \\
\hline $\mathbf{m}$ & $\mathbf{n}$ & $\mathbf{M H z}$ & yes/no \\
\hline 0 & 0 & - & no \\
\hline 1 & 0 & 938 & yes \\
\hline 0 & 1 & 1250 & yes \\
\hline 1 & 1 & 1563 & yes \\
\hline
\end{tabular}

Table I and Table II show that the resonance frequencies of the cavity field depend significantly on the boundary conditions. Equation (6) offers a quick opportunity to estimate the resonance frequencies of a slim enclosure.

We derived the impedance matrix for the consideration of the external environment of the enclosure by using the equivalent source method, similarly as it has been done in [3] for planes with open boundaries. In case of an enclosure the metallic walls influence the field distribution. For the exact consideration of this influence a boundary value problem for 
the exterior of the enclosure has to be solved by a field simulation program. To obtain an efficient design formulation, we neglected the influence of the metallic walls and obtained the far field formulations only from the slot fields. We compared the radiated power calculated with this formulation with three-dimensional full wave calculations using $\mathrm{HFSS}^{\circledR}$, which showed that the contribution of the enclosure walls to the radiated power can be neglected. Therefore the presented far field expressions can be used for the efficient calculation of the radiated power and the impedance matrix for the consideration of the exterior of the enclosure. Equivalent sources at the slot lead to the following integrals for the far field (Coordinate system B Fig. 1):

$$
\begin{aligned}
& \vec{E} \approx-\frac{j \cdot k}{4 \cdot \pi} \cdot \frac{e^{-j \cdot k \cdot r}}{r} \cdot \sin (\vartheta) \cdot \vec{e}_{\varphi} \\
& \int_{z=0}^{L} U(z) \cdot e^{j \cdot k \cdot z \cdot \cos (\vartheta)} \cdot d z
\end{aligned}
$$

The radiated power density in the far field is given by:

$$
\vec{S}=\vec{E} \times \vec{H}^{*}
$$

Expression (9) can be used to define an admittance network (matrix), which can be connected to ports at the slot of the enclosure for the consideration of the radiation loss.

$$
\begin{aligned}
& Y_{a_{-} z s} \approx \frac{S_{z s}}{U_{z} \cdot U_{s}^{*}}=\frac{k^{3}}{8 \cdot \pi \cdot \omega \cdot \mu_{0}} \cdot\left(\frac{L}{n_{-} \text {port }}\right)^{2} \\
& \cdot \int_{\vartheta=0}^{\pi} \sin ^{3}(\vartheta) \cdot e^{j \cdot k \cdot\left(z_{z}-z_{s}\right) \cdot \cos (\vartheta)} \cdot d \vartheta \\
& \mathrm{z}_{\mathrm{z}}, \mathrm{z}_{\mathrm{s}} \ldots \text { port positions at the open slot }
\end{aligned}
$$

The number of ports $n \_$port which is necessary to achieve a dedicated accuracy depends on the maximum frequency. A calculation with an increased number of ports can be performed to check, whether $n \_$port is sufficiently high.

The cavity fields with radiation loss are calculated with (2) and (4) :

$$
\left(\begin{array}{l}
U_{m} \\
U_{S} \\
U_{p 1} \\
\vdots \\
U_{p n}
\end{array}\right)=\left(\begin{array}{ccccc}
0 & Z_{m s} & Z_{m p 1} & \cdots & Z_{m p n} \\
0 & Z_{s s} & Z_{s p 1} & \cdots & Z_{s p n} \\
0 & Z_{p 1 s} & Z_{p 1 p 1} & \cdots & Z_{p 1 p n} \\
\vdots & \vdots & \vdots & & \vdots \\
0 & Z_{p n s} & Z_{p n p 1} & \cdots & Z_{p n p n}
\end{array}\right) \cdot\left(\begin{array}{l}
I_{m} \\
I_{S} \\
I_{p 1} \\
\vdots \\
I_{p n}
\end{array}\right)
$$

$\mathrm{U}_{\mathrm{m}} \quad$ port voltage at the measurement port (at the slot).

$\mathrm{U}_{\mathrm{S}} \quad$ port voltage at the source port inside the enclosure.

$\mathrm{U}_{\mathrm{pi}} \quad$ port voltages of the interface ports at the slot.

$\mathrm{I}_{\mathrm{m}} \quad$ port current at the measurement port (at the slot).

$\mathrm{I}_{\mathrm{S}} \quad$ port current at the source port inside the enclosure.

$\mathrm{I}_{\mathrm{pi}} \quad$ port currents of the interface ports at the slot.

Matrix notation gives:

$$
\begin{aligned}
& \left(\begin{array}{l}
U_{p 1} \\
\vdots \\
U_{p n}
\end{array}\right)=\left(\begin{array}{l}
Z_{p 1 s} \\
\vdots \\
Z_{p n s}
\end{array}\right) \cdot I_{s}+\left(\begin{array}{ccc}
Z_{p 1 p 1} & \cdots & Z_{p 1 p n} \\
\vdots & & \vdots \\
Z_{p n p 1} & \cdots & Z_{p n p n}
\end{array}\right) \cdot\left(\begin{array}{l}
I_{p 1} \\
\vdots \\
I_{p n}
\end{array}\right) \\
& \underline{U}_{p}=\underline{Z}_{p s} \cdot \underline{I}_{s}+\underline{Z}_{p p} \cdot \underline{I}_{p}
\end{aligned}
$$

$\underline{U}_{m}=\underline{Z}_{m s} \cdot \underline{I}_{s}+\underline{Z}_{m p} \cdot \underline{I}_{p}$

The relation of the voltage vector $\mathrm{U}_{\mathrm{p}}$ and the current vector $I_{p}$ at the interface ports is given by the admittance matrix (10):

$$
\underline{U}_{p}=-\left(Y_{a}\right)^{-1} \cdot \underline{I}_{p}=-Z_{a} \cdot \underline{I}_{p}
$$

$Z_{a}$ is the impedance matrix used to introduce the influence of the external environment to the cavity formulation (11). This leads to the final formulation for the voltage on the measurement port:

$$
\underline{U}_{m}=\left(\underline{Z}_{m s}-\underline{Z}_{m p} \cdot\left(\underline{Z}_{p p}+Z_{a}\right)^{-1} \cdot \underline{Z}_{p s}\right) \cdot \underline{I}_{s}
$$

and the port voltages on the interface:

$$
\underline{U}_{p}=-\left(Y_{a}\right)^{-1} \cdot\left(\underline{Z}_{p p}+Z_{a}\right)^{-1} \cdot \underline{Z}_{p s} \cdot \underline{I}_{s}
$$

The radiation loss is very low below the first resonance frequency. Therefore, $Y_{a}$ is nearly singular for lower frequencies and (15) (16) cannot be evaluated in this frequency range. There, the radiation loss can be neglected and (15) (16) can be simplified for frequencies below the first resonance to:

$$
\underline{U}_{m}=\underline{Z}_{m s} \cdot \underline{I}_{s}
$$




$$
\underline{U}_{p}=\underline{Z}_{p s} \cdot \underline{I}_{s}
$$

The voltages on the slot are given by (15) to (18) and the currents of all ports can be calculated straightforwardly.

(8) can be solved by use of (2):

$$
\begin{aligned}
& \vec{E} \approx \frac{k \cdot \omega \cdot \mu_{0} \cdot h}{\pi \cdot W \cdot L} \cdot \frac{e^{-j \cdot k \cdot r}}{r} \cdot I_{S} \cdot \sin (\vartheta) \\
& \left\{\sum _ { m = 0 } ^ { \infty } \sum _ { n = 0 } ^ { \infty } \left[\frac{\sin \left(k_{m} \cdot z_{S}\right) \cdot \sin \left(k_{n} \cdot\left(x_{S}+W\right)\right)}{k_{m}^{2}+k_{n}^{2}-k^{2}}\right.\right. \\
& \left.\left.(-1)^{n} \cdot \frac{k_{m}}{k_{m}^{2}-k^{2} \cdot \cos ^{2}(\vartheta)} \cdot\left(1-(-1)^{m} \cdot e^{j \cdot k \cdot L \cdot \cos (\vartheta)}\right)\right]\right\} \cdot \vec{e}_{\varphi}
\end{aligned}
$$

With (19) the radiated power from the slot can be expressed by a closed form expression:

$$
\vec{S} \approx \frac{k}{\omega \cdot \mu} \cdot|\vec{E}|^{2} \cdot \vec{e}_{r}
$$

\section{COMPARISON WITH HFSS AND APPLICATION FOR PLACEMENT INVETSIGATIONS}

The transfer impedance $\mathrm{Z}_{\mathrm{t}}=\mathrm{Up}_{\mathrm{M}} / \mathrm{Ip}_{\mathrm{S}}$ of the current on source port $\mathrm{p}_{\mathrm{S}}$ at position $\mathrm{x}_{\mathrm{S}}=\mathrm{L} / 2, \mathrm{y}_{\mathrm{S}}=50 \mathrm{~mm}$ to the measurement port $\mathrm{p}_{\mathrm{M}}$ at position $\mathrm{x}_{\mathrm{S}}=\mathrm{L} / 2, \mathrm{y}_{\mathrm{S}}=\mathrm{W}$ has been calculated with $\mathrm{HFSS}^{\circledR}$ and with the cavity model. The dimensions of the enclosure are $\mathrm{L}=134 \mathrm{~mm}, \mathrm{~W}=104 \mathrm{~mm}$ and $\mathrm{h}=7 \mathrm{~mm}$ (Coordinate system A).

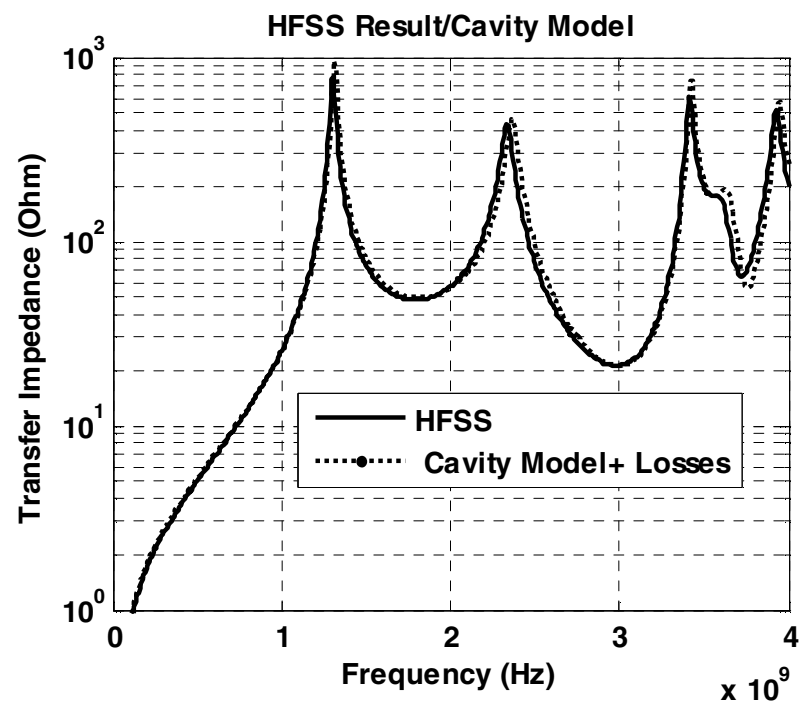

Figure 3. Comparison of the HFSS ${ }^{\circledR}$ simulation result to the cavity model result. The external environment of the enclosure has been taken into account by the connection of the impedance network to the interface ports at the slot.
Fig. 3 shows a good agreement between the $\mathrm{HFSS}^{\circledR}$ result and the cavity model, especially at the resonance frequencies, where the radiation emission is high. Therefore the radiation loss has been introduced correctly by the impedance network, which has been connected to the interface ports at the slot to consider the external environment. Fig. 4 shows the comparison of the $\mathrm{HFSS}^{\circledR}$ result with the cavity model without consideration of the radiation loss:

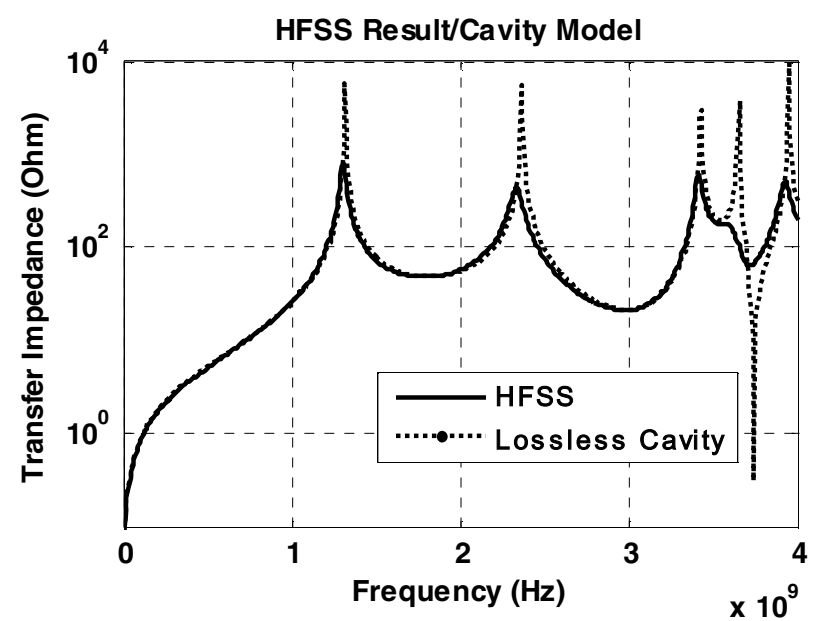

Figure 4. Comparison of the $\operatorname{HFSS}^{\circledR}$ simulation result to the cavity model result. The external environment of the enclosure has not been taken into account. The cavity model is lossless.

The comparison in Fig. 4 shows that the radiation loss has a significant influence especially at the resonance frequencies. It has to be taken into account for the internal solution and also for the calculation of the radiated emission. Fig. 3 and Fig. 4 give also evidence that the expression for the radiated power (20), which was obtained without the consideration of the influence from the enclosure walls, is sufficient up to high enclosure resonance modes.

We used the cavity model for the presentation of the separation method, because we wanted to show that the radiation loss can be considered in cavity models by interface ports at the cavity boundaries. Cavity models are frequently used for power network design [1] [2] and the radiated emission has an influence on the internal cavity solution, when the separation distance of the planes increases [5]. In such a case the radiation loss has to be taken into account. Our separation method provides an opportunity to consider the radiation loss with an impedance network, connected to interface ports at the cavity boundaries. This network is independent of the internal solution and once calculated for a given cavity shape it can be used for any source configuration inside the power planes or a slim enclosure.

However, the separation method is not limited to this application. It can be used with simulation tools like $\mathrm{HFSS}^{\circledR}$ for separately simulating the exterior and the interior of devices with arbitrary shape and slim slots. The method can also be used to connect models which have been established with different simulation programs. 
As an application of the cavity model we use the power calculation to investigate the influence of the source position on the radiated power from the slot of the enclosure. Radiated emission initiated by a single source can be significantly reduced, when the source is moved closer to a wall of the enclosure. As an example we present the radiated power of an enclosure with $\mathrm{L}=160 \mathrm{~mm}, \mathrm{~W}=120 \mathrm{~mm}$ and $\mathrm{h}=7 \mathrm{~mm}$ and varying source positions (Fig. 5):

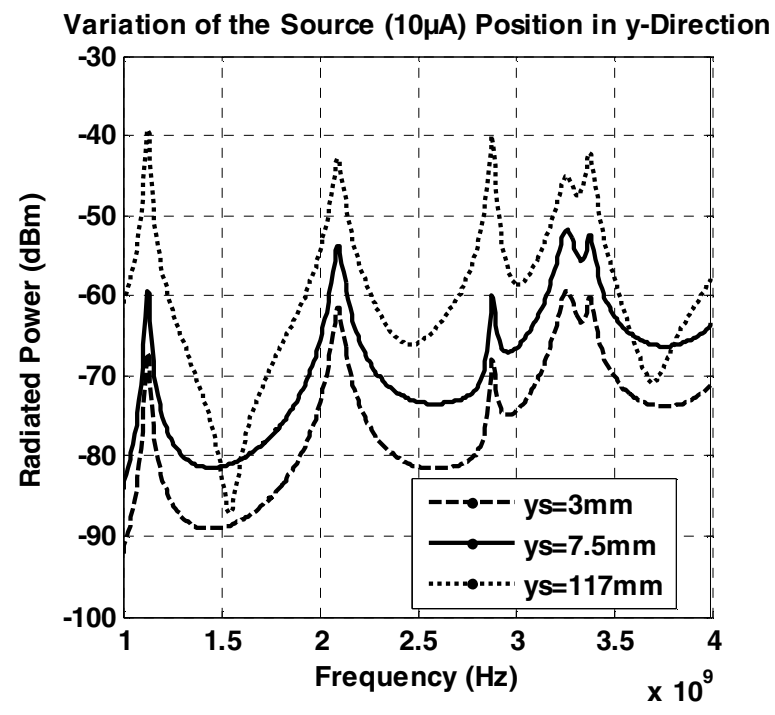

Figure 5. Variation of the excitation source position ( $\mathrm{xs}=80 \mathrm{~mm})$. The radiated power decreases, when the source is placed near the rear wall. The coordinates (xs, ys) correspond to the Coordinate system A in Fig. 1.

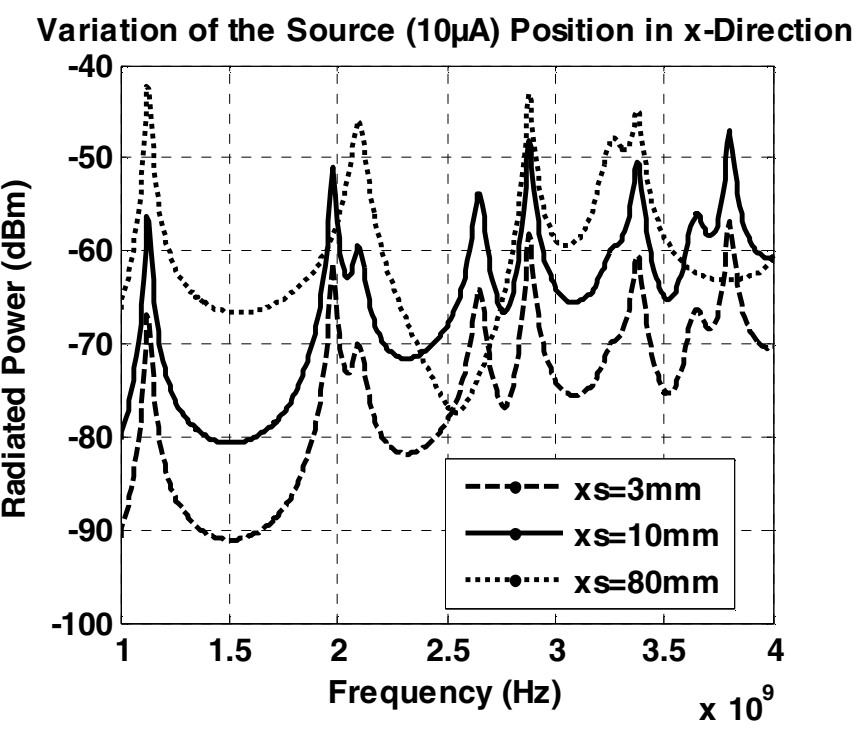

Figure. 6 Variation of the excitation source position $(\mathrm{ys}=60 \mathrm{~mm})$. The radiated power decreases, when the source is placed a near a side wall. The Coordinates (xs, ys) corresponding to Coordinate system A in Fig. 1.
The $\sin ()$ functions in (2), which depend on the source position vanish at the enclosure walls. Therefore, the cavity field inside the enclosure is reduced, when a source is placed closer to a wall. This results in a lower radiation from the slot. Each placement of a single source closer to the enclosure walls will reduce the emissions from this source. As shown in this example, our method is suitable for placement investigations. It is not restricted to only one source. (2) and (19) can be used to calculate the field from multiple sources, because these equations are linear and the contributions of multiple sources can be superposed. Afterwards the radiated power can be evaluated using (20).

\section{CONCLUSIONS}

We presented a domain separation method with port interfaces for the separate simulation of the interior and the external environment of enclosures with slots. The method can be used with any simulation environment, where it is possible to define PEM boundary conditions and port excitations. As an example we mentioned HFSS ${ }^{\circledR}$. However, we used the method also with the analytical cavity calculation method.

For a slim metallic enclosure with three closed walls and one open edge, we presented an analytical cavity model. This model can be used for efficient design investigations. The placement influence on the radiated emission from critical electronic devices within the enclosure can be evaluated with our method.

\section{ACKNOWLEDGMENT}

This work was supported by the Medea+ Parachute program.

\section{REFERENCES}

[1] G.-T. Lei, R. W. Techentin, and B. K. Gilbert, "High-Frequency Characterization of Power/Ground-Plane Structures", IEEE Trans. Microw. Theory Tech., Vol. 47 No. 5, May 1999, pp.562-569

[2] C. Wang, J. Mao, G. Selli, S. Luan, L. Zhang, J. Fan, D. J. Pommerenke, R. E. DuBroff, J. L. Drewniak, "An Efficient Approach for Power Delivery Network Design with Closed-Form Expressions for Parasitic Interconnect Inductances", IEEE Trans. of Adv. Packaging, Vol.29, No.2, May 2006, pp.320-334

[3] Marco Leone, "The Radiation of a Rectangular Power-Bus Structure at Multiple Cavity-Mode Resonances", IEEE Trans. on Electromagnetic Compatibility, Vol. 45, No.3, August 2003, pp .486-492

[4] Richard L. Chen, Ji Chen, Todd H. Hubing, Weimin Shi, "Analytical Model for the Ractangular Power-Ground Structure Including Radiation Loss.”, IEEE Trans. on Electromagnetic Compatibility, Vol. 47, No. 1, February 2005, pp. 10-16

[5] Richard L. Chen, Ji Chen, Todd H. Hubing, Weimin Shi, "Via Coupling within Power-Return Plane Structures Considering the Radiation Loss", International Symposium on Electromagnetic Compatibility 2004, EMC 2004, Vol.2, August 2004, pp.386-391

[6] Hwan-Woo Shim, Todd H. Hubing, "A Closed-Form Expression for Estimating Radiated Emissions From the Power Planes in a Populated Printed Circuit Board", IEEE Trans. on Electromagnetic Compatibility, Vol.48, No. 1, February 2006, pp. 74-81

[7] W. L. Stutzmann and G. A. Thiele, Antenna Theory and Design. New York: Wiley 